\title{
Diagramas de fase CVD para la preparación de películas de iridio(•)
}

\author{
M.A. Hernández-Pérez*, J.R. Vargas-García* y J.A. Romero-Serrano*
}

Resumen

\begin{abstract}
Se calcularon los diagramas de fase CVD (Chemical Vapor Deposition) para la preparación de películas de iridio empleando el método de minimización de la energía libre de Gibbs. Como precursor se utilizó acetilacetonato de iridio $\left(\operatorname{Ir}(\mathrm{acac})_{3}\right)$. Se analizaron las mezclas gaseosas $\operatorname{Ir}(\mathrm{acac})_{3}-\mathrm{O}_{2}$-Ar e $\operatorname{Ir}(\mathrm{acac})_{3}$-Ar. Las temperaturas de depósito se exploraron desde 300 hasta $800{ }^{\circ} \mathrm{C}$, las presiones totales de 13,3 a $13.332 \mathrm{~Pa}$ y las presiones parciales de los gases $\operatorname{Ir}(\mathrm{acac})_{3}$ y $\mathrm{O}_{2}$ desde 0,001 hasta $1.000 \mathrm{~Pa}$. Los diagramas $\mathrm{Ir}-\mathrm{CVD}$ predicen que $\sin \mathrm{O}_{2}$ en la mezcla gaseosa, las películas constan de las fases sólidas Ir+C. En contraste, con adición de $\mathrm{O}_{2}$ los diagramas Ir-CVD revelan diferentes dominios de fases sólidas que incluyen $\mathrm{IrO}_{2}, \mathrm{IrO}_{2}+\mathrm{Ir}$, $\mathrm{Ir}$ e $\mathrm{Ir}+\mathrm{C}$. Estos diagramas permiten establecer las presiones totales y temperaturas requeridas para obtener películas de composición deseada. Los resultados predichos por los diagramas Ir-CVD, están en buena concordancia con los obtenidos experimentalmente.
\end{abstract}

Palabras clave Iridio. Películas delgadas. Diagramas de fase CVD.

\section{CVD phase diagrams for iridium films preparation}

\begin{abstract}
Chemical vapor deposition (CVD) phase diagrams for the preparation of iridium films were calculated using Gibbs free energy minimization method. Iridium acetylacetonate $\left(\operatorname{Ir}(\mathrm{acac})_{3}\right)$ was used as the precursor compound. Two gaseous mixtures were analyzed: $\operatorname{Ir}(\mathrm{acac})_{3}-\mathrm{O}_{2}$ - $\mathrm{Ar}$ and $\operatorname{Ir}(\mathrm{acac})_{3}$ - Ar. The deposition temperatures were explored from 300 to $800{ }^{\circ} \mathrm{C}$, total pressures from 13.3 to $13.332 \mathrm{~Pa}$ and partial pressures of $\operatorname{Ir}(\mathrm{acac})_{3}$ gas and $\mathrm{O}_{2}$ gas from 0.001 to $1.000 \mathrm{~Pa}$. The Ir-CVD diagrams predicted that without $\mathrm{O}_{2}$ gas in the gaseous mixture, the solid films consist of two solid phases: $\mathrm{Ir}+\mathrm{C}$. In contrast, with addition of $\mathrm{O}_{2}$ to the gaseous mixture, the $\mathrm{Ir}-\mathrm{CVD}$ diagrams revealed different domains of condensed phases which include $\mathrm{IrO}_{2}, \mathrm{IrO}_{2}+\mathrm{Ir}, \mathrm{Ir}$ and $\mathrm{Ir}+\mathrm{C}$. These diagrams allow one to establish the total pressures and temperatures required to obtain a given film composition. The results predicted by the Ir-CVD diagrams are in good agreement with those experimentally obtained.
\end{abstract}

Keywords Iridium. Thin films. CVD phase diagrams.

\section{INTRODUCCIÓN}

En años recientes, las películas delgadas de metales del grupo platino han recibido especial atención tanto por su aplicación como contactos para circuitos electrónicos y depósitos de combustible como en la preparación de catalizadores de alta eficiencia debido a la buena conductividad eléctrica, alta estabilidad química y actividad catalítica que presentan estos metales ${ }^{[1-7]}$. La composición química, morfología y estructura de las películas tienen un impacto significativo sobre sus propiedades, las cuales son decisivas para su aplicación en particular. Entre las técnicas disponibles para la preparación de películas delgadas, la técnica chemical vapor deposition (CVD), brinda ventajas para lograr los requerimientos estructurales y de composición, debido a la facilidad de control de las variables de operación ${ }^{[8]}$. A pesar de que CVD es una técnica atractiva, generalmente es difícil optimizar las variables del proceso. Frecuentemente es necesario un gran número de experimentos para determinar las condiciones

(•) Trabajo recibido el día 19 de marzo de 2001 y aceptado en su forma final el día 20 de septiembre de 2001.

$(*) \quad$ Dpto. de Ingeniería Metalúrgica, ESIQIE-IPN, A.P. 75-874, México D.F. 
óptimas de operación, como temperatura de depósito, presión total y presiones parciales de las diferentes especies gaseosas, entre otras. A menos que haya un trabajo de experimentación extenso, los resultados son, comunmente, insatisfactorios. Un camino más eficiente para la aplicación de la técnica CVD, mejor que prueba y error, comienza con un análisis termodinámico para establecer los efectos de los parámetros de depósito en la composición, morfología y estructura de las películas. En particular, los diagramas de fase CVD construídos a partir de las funciones termodinámicas de las sustancias involucradas en el proceso son de gran utilidad, ya que pueden predecir fácilmente la naturaleza de las fases depositadas como una función de los parámetros experimentales, tales como las presiones parciales de los gases precursor, de reacción y de arrastre, a condiciones de presión total y temperaturas definidas ${ }^{[9]}$. Las investigaciones para la preparación de películas de iridio (Ir) por CVD, han sido orientadas a la determinación experimental de los parámetros de depósito, utilizando una amplia variedad de compuestos metal-orgánicos como precursores de Ir [10-12]. Como una etapa inicial en la simulación de la técnica CVD o metal organic chemical vapor deposition (MOCVD) para Ir, en este trabajo, se proporcionan los diagramas CVD para el depósito de películas de Ir, cuando se utiliza el compuesto precursor metal-orgánico, acetilacetonato de $\operatorname{Ir}\left(\operatorname{Ir}(\mathrm{acac})_{3}\right)$ y se comparan con resultados experimentales obtenidos en trabajos previos ${ }^{[6,7}$ y 12$]$.

\section{TÉCNICA DE CÁLCULO}

\subsection{Cálculo de los diagramas de fase Ir-CVD}

La descomposición térmica del acetilacetonato de Iridio $\operatorname{Ir}(\mathrm{acac})_{3}\left(\left(\mathrm{CH}_{3}-\mathrm{COCHCO}-\mathrm{CH}_{3}\right)_{3} \mathrm{Ir}\right)$ en el proceso LP-MOCVD (low pressure MOCVD) genera un sistema químico heterogéneo complejo, con un gran número de especies gaseosas y con, al menos, dos especies sólidas: iridio (Ir) y carbono (C). Para la determinación de las especies químicas en equilibrio con participación significativa en la formación de películas de Ir, se utilizó el programa Equilib, el cual forma parte del sistema de análisis termodinámico FACT-Win ${ }^{[13]}$. El programa Equilib se basa en el método de minimización de la energía libre de Gibbs. Este programa proporciona las cantidades de las especies químicas en equilibrio, bajo las condiciones de presión total, temperatura y cantidades de reactivos especificadas.
El método de minimización de energía libre se basa en el hecho de que la energía libre de Gibbs total de un sistema en equilibrio, tiene su valor mínimo de acuerdo a la expresión:

$$
\left(\frac{\partial G}{\partial n_{i}}\right)_{n_{j}}=0
$$

donde $\mathrm{n}_{\mathrm{i}}$ es el número de moles de un componente del sistema. En general, la energía libre total de un sistema a temperatura y presión constantes dependerá de las cantidades de todos los componentes en las diferentes fases. En un sistema dado, las especies pueden formar parte de soluciones químicas (sólidas, líquidas o gaseosas) o estar en estado puro. La energía libre total del sistema puede expresarse en términos de las energías libres molares parciales, o potenciales químicos, y del número de moles de cada componente mediante:

$$
\mathrm{G}=\sum \mathrm{n}_{\mathrm{i}} \mathrm{g}_{\mathrm{i}}
$$

donde $\mathrm{g}_{\mathrm{i}}$ es la energía libre molar parcial de la especie $i$, la cual se relaciona con la actividad mediante $\mathrm{g}_{\mathrm{i}}=\mathrm{g}_{\mathrm{i}}^{\mathrm{o}}+\mathrm{RT} \ln \left(a_{i}\right)$.

Evidentemente, si la especie es pura, el potencial químico será exactamente igual a su energía libre molar estándar: $g_{i}=g_{i}^{o}$. La expresión general de energía libre total de cualquier sistema se puede expresar como:

$$
\begin{aligned}
& \mathrm{G}=\sum \mathrm{n}_{\mathrm{i}}^{\mathrm{g}} \mathrm{g}_{\mathrm{i}}^{\mathrm{o}, \mathrm{g}}+\mathrm{RT} \sum \mathrm{n}_{\mathrm{i}}^{\mathrm{g}} \ln \left(\mathrm{p}_{\mathrm{i}}^{\mathrm{g}}\right)+\sum \mathrm{n}_{\mathrm{i}}^{1} \mathrm{~g}_{\mathrm{i}}^{\mathrm{o}, l}+ \\
& + \text { RT } \sum \mathrm{n}_{\mathrm{i}}^{l} \ln \left(a_{i}^{l}\right)+\sum \mathrm{n}_{\mathrm{i}}^{\mathrm{s}} \mathrm{g}_{\mathrm{i}}^{\mathrm{o}, \mathrm{s}}+\mathrm{RT} \sum \mathrm{n}_{\mathrm{i}}^{\mathrm{s}} \ln \left(a_{\mathrm{i}}^{\mathrm{s}}\right)
\end{aligned}
$$

donde los superíndices $\mathrm{g}, 1$ y s denotan las fases gas, líquido y sólido, respectivamente; $\mathrm{p}_{\mathrm{i}}^{\mathrm{g}}$ es la presión parcial de un componente en la fase gaseosa.

La disponibilidad de datos termodinámicos de los productos de la descomposición térmica del $\operatorname{Ir}(\mathrm{acac})_{3}$ es limitada. Consecuentemente, las especies químicas consideradas en el cálculo del equilibrio químico complejo, en este trabajo, incluyeron los productos gaseosos significativos de la descomposición de varios acetilacetonatos metálicos reflejados en la literatura ${ }^{[14-16]}$. La tabla I resume las especies químicas consideradas en el cálculo termodinámico.

Para el cálculo de los diagramas Ir-CVD se consideró un sistema cerrado en el que la fase gaseosa 
Tabla I. Especies químicas consideradas en el análisis termodinámico

Table I. Chemical species considered for the thermodynamical analysis

\begin{tabular}{|c|c|}
\hline Fase & Especie \\
\hline \multirow[t]{13}{*}{ Gas ideal } & $\operatorname{Ir}(\mathrm{acac})_{3}$ \\
\hline & acac \\
\hline & $\mathrm{Ar}$ \\
\hline & $\mathrm{CO}$ \\
\hline & $\mathrm{CO}_{2}$ \\
\hline & $\mathrm{H}_{2} \mathrm{O}$ \\
\hline & $\mathrm{CH}_{4}$ \\
\hline & $\mathrm{CH}_{3} \mathrm{COCH}_{3}$ \\
\hline & $\mathrm{CH}_{2} \mathrm{CO}$ \\
\hline & $\mathrm{CH}_{3} \mathrm{COCHCO}$ \\
\hline & $\mathrm{CH}_{3} \mathrm{COOH}$ \\
\hline & $\mathrm{CH}_{3} \mathrm{COCH}_{2} \mathrm{CH}_{3}$ \\
\hline & $\mathrm{IrO}_{3}$ \\
\hline \multirow[t]{3}{*}{ Sólido puro } & Ir \\
\hline & $\mathrm{IrO}_{2}$ \\
\hline & $\mathrm{C}$ \\
\hline
\end{tabular}

es una solución ideal y los sólidos son especies puras. En base a las cantidades en equilibrio de las fases gaseosa y sólida resultantes del cálculo, se construyeron los diagramas Ir-CVD. Las condiciones exploradas fueron: temperatura de depósito $\left(\mathrm{T}_{\text {dep }}\right)$ entre 300 y $800{ }^{\circ} \mathrm{C}$; presiones totales $\left(\mathrm{P}_{\text {tot }}\right)$ entre 13,3 y $13.332 \mathrm{~Pa}$; presiones parciales de $\operatorname{Ir}(\mathrm{acac})_{3}$ y $\mathrm{O}_{2}\left(P_{\mathrm{Ir}(\text { acac })_{3}}, P_{\mathrm{O}_{2}}\right)$ entre 0,01 y $1.000 \mathrm{~Pa}$. Con estos parámetros se generaron 24 diagramas Ir-CVD.

En el cálculo de los diagramas se consideraron dos mezclas gaseosas de reactivos de alimentación a la cámara del reactor Ir-CVD: la primera mezcla constituida únicamente por $\operatorname{Ir}(\mathrm{acac})_{3}$-Ar y la segunda incluyendo oxígeno $\operatorname{Ir}(\mathrm{acac})_{3}-\mathrm{O}_{2}$ - Ar.

\subsection{Estimación de datos termodinámicos}

Los datos termodinámicos de la mayoría de las especies se obtuvieron de la base de datos del sistema FACT-Win. Sin embargo, los datos para las especies $\operatorname{Ir}\left(\right.$ acac) ${ }_{3}$, acetilacetona (acac) y $\mathrm{CH}_{3} \mathrm{COCH}$ $\mathrm{CO}$, tuvieron que calcularse y anexarse a una base de datos privada. La entalpía de formación estándar $\left(\Delta \mathrm{H}^{\circ}\right)$ y la entropía estándar $\left(\mathrm{S}^{\circ}\right)$ de las especies mencionadas, se calcularon con ayuda del método de contribución de grupos de Benson ${ }^{[17]}$. Particularmente, el valor $\Delta \mathrm{H}^{\circ}$ de acac se obtuvo del trabajo experimental de Hacking y Pilcher ${ }^{[18]}$.
La estimación de la capacidad calorífica $\left(\mathrm{Cp}^{\circ}\right)$ se efectúo con la ecuación de Dobratz (4) en conjunto con las modificaciones de Meghreblian ${ }^{[19]}$. La estimación se basa en las frecuencias vibracionales de estiramiento y flexión $(v, \delta)$, características de los diferentes tipos de enlaces de una molécula. Esto lleva a la ecuación típica de capacidad calorífica en función de la temperatura (ec. 9) resultante del análisis de Crawford y $\operatorname{Parr}^{[19}$ y 20$]$.

$$
\begin{gathered}
C p^{o}=4 R+m(2 / R)+\sum q_{i} C_{v i}+ \\
+\left[\left(3 n-6-m-\sum q_{i}\right) / \sum q_{i}\right]\left[\sum q_{i} C_{\delta i}\right]
\end{gathered}
$$

donde:

$\mathrm{Cp}^{\circ}=$ capacidad calorífica del gas $(\mathrm{J} / \mathrm{gmol} \mathrm{K})$

$\mathrm{R}=$ constante de los gases ideales (J/gmol K)

$\mathrm{m}=$ número de enlaces simples donde los grupos puedan sufrir rotación interna (p.e.: C-C, C-O en ésteres y éteres)

$\mathrm{q}_{\mathrm{i}}=$ número de enlaces del tipo $\mathrm{i}$

$\mathrm{n}=$ número de átomos en la molécula

$\Sigma \mathrm{q}_{\mathrm{i}}=$ número total de enlaces en la molécula

$\mathrm{C}_{\mathrm{vi}}, \mathrm{C}_{\delta \mathrm{i}}=$ funciones de Einstein para enlaces del tipo i, es decir, $\mathrm{R}\left(\mathrm{X}^{2}\right) e^{\mathrm{X}}\left(e^{\mathrm{X}-1}\right)^{2}$

$X=h v / k T$ para $C_{v i}$ y $X=h \delta / k T$ para $C_{\delta i}$

$\mathrm{h}=$ constante de Planck, es decir, 6,61 $\times 10^{-34}$ $\mathrm{J}$-s/mol

$v=$ frecuencia característica para vibraciones longitudinales, $\mathrm{s}^{-1}$

$\delta=$ frecuencia característica para vibraciones transversales, $\mathrm{s}^{-1}$

$\mathrm{k}=$ constante de Boltzman, es decir, $13,77 \times 10^{-24}$ $\mathrm{J} / \mathrm{mol} \mathrm{K}$

$\mathrm{T}=$ temperatura, $\mathrm{K}$

La frecuencia $(v)$ se relaciona con la longitud de onda $(\lambda)$ y el número de onda $(\bar{v})$ en la forma:

$$
v=\mathrm{c} \lambda=\mathrm{c} \bar{v}
$$

donde: $\mathrm{c}=$ velocidad de la luz, $3 \times 10^{8} \mathrm{~m} / \mathrm{s}$

El parámetro X se escribe entonces como:

$$
\mathrm{X}=\mathrm{h} \bar{v} \mathrm{c} / \mathrm{kT}
$$

Considerando que los términos $\mathrm{h}, \mathrm{c}$ y $\mathrm{k}$ son constantes: 


$$
\mathrm{X}=1.4385 \bar{v} / \mathrm{T}
$$

donde: $\bar{v}=$ número de onda característico para vibraciones de estiramiento $\left(\bar{v}_{v}\right)$ y flexión $\left(\bar{v}_{\delta}\right)$ de enlaces del tipo i.

Los valores de números de onda de vibración para diversos enlaces de una molécula se encuentran tabulados de acuerdo a Dobratz ${ }^{[19]}$. Estos hacen posible la estimación de los parámetros: $\mathrm{X}, \mathrm{C}_{\mathrm{vi}}$ y $\mathrm{C}_{\delta \mathrm{i}}$ y en consecuencia la estimación del valor $\mathrm{Cp}^{\circ}$ a través de la ecuación (4). Por otra parte, también es posible estimar el valor Cp utilizando la serie propuesta por Dobratz que incluye las constantes $A_{i}, B_{i}$ y $C_{i}$ (ec. 8).

$$
\mathrm{C}_{v i} \circ \mathrm{C}_{\delta \mathrm{i}}=\mathrm{A}_{\mathrm{i}}+\mathrm{B}_{\mathrm{i}} \mathrm{T}+\mathrm{C}_{\mathrm{i}} \mathrm{T}^{2}
$$

Las modificaciones propuestas por Meghreblian, Crawford y Parr a los valores dados por Dobratz, llevan a la ecuación típica de la capacidad calorífica en función de la temperatura de la forma:

$$
\mathrm{Cp}^{\circ}=\mathrm{A}+\mathrm{BT}+\mathrm{CT}^{2}
$$

donde:

$\mathrm{Cp}^{\circ}=$ capacidad calorífica del gas o vapor a baja presión $\left(\mathrm{J} \mathrm{gmol}^{-1} \mathrm{~K}^{-1}\right)$

$\mathrm{A}, \mathrm{B}$ y $\mathrm{C}=$ constantes resultantes de la ecuación de Dobratz

$\mathrm{T}=$ temperatura $(\mathrm{K})$

Este método tiene buena aproximación en la determinación de capacidades caloríficas de gases y vapores puros a baja presión ${ }^{[20]}$.

\subsection{Determinación experimental de la compo- sición de las mezclas gaseosas de alimen- tación}

Las fracciones molares de las especies en las mezclas gaseosas $\operatorname{Ir}(\mathrm{acac})_{3}-\mathrm{O}_{2}$ - $\mathrm{Ar}$ y $\operatorname{Ir}(\mathrm{acac})_{3}$ - Ar se estimaron de acuerdo a sus flujos molares alimentados a un reactor horizontal LP-MOCVD de pared caliente $(0,05 \mathrm{~m}$ de diámetro y $1,2 \mathrm{~m}$ de longitud). Los flujos volumétricos de $\mathrm{Ar}$ y $\mathrm{O}_{2}$ se controlaron bajo condiciones de presión y temperatura ambientales ( $78 \mathrm{KPa}$ y $25^{\circ} \mathrm{C}$ ). El flujo volumétrico de $\operatorname{Ar}$ se mantuvo constante en $8,33 \times 10^{-7} \mathrm{~m}^{3} / \mathrm{s}$. Su flujo molar se calculó en $2,62 \times 10^{-5} \mathrm{gmol} / \mathrm{s}$ debido a que su factor de compresibilidad $(z)$ a 78 $\mathrm{KPa}$ y $25^{\circ} \mathrm{C}$ es igual a $\mathrm{z}=0,999546$. El flujo volumétrico de $\mathrm{O}_{2}$ máximo en la mezcla gaseosa $\operatorname{Ir}(\mathrm{acac})_{3}-\mathrm{O}_{2}$-Ar fue de $5 \times 10^{-8} \mathrm{~m}^{3} / \mathrm{s}$ al que corresponde un flujo molar máximo de $1,58 \times 10^{-6}$ $\mathrm{gmol} / \mathrm{s}(z=0,998893)$. El flujo molar de $\operatorname{Ir}(\mathrm{acac})_{3}$ se estimó en $4 \times 10^{-7} \mathrm{gmol} / \mathrm{s}$ con base en el tiempo de evaporación de una cantidad conocida $(5 \times$ $10^{-2} \mathrm{~g}$ ) a $\mathrm{P}_{\text {tot }}=13,3$ Pa y $180^{\circ} \mathrm{C}$. Dado que la velocidad de evaporación es inversamente proporcional a la presión total, el flujo molar de $\operatorname{Ir}(\mathrm{acac})_{3}$ se consideró máximo. La tabla II resume las fracciones molares de las especies químicas que forman la mezcla gaseosa de alimentación $\operatorname{Ir}(\mathrm{acac})_{3}-\mathrm{O}_{2}-\mathrm{Ar}$.

\section{RESULTADOS Y DISCUSIÓN}

La figura 1 muestra los diagramas de fase Ir-CVD a diferentes temperaturas de depósito para la mezcla gaseosa $\mathrm{Ir}(\mathrm{acac})_{3}-\mathrm{O}_{2}-\mathrm{Ar}$, en función de las presiones parciales de $\operatorname{Ir}(\mathrm{acac})_{3}$, y $\mathrm{O}_{2}$ a una presión total de $133,3 \mathrm{~Pa}$. La figura $1 \mathrm{a}$ ), a $\mathrm{T}_{\text {dep }}=300^{\circ} \mathrm{C}$, muestra que la fase sólida $\mathrm{IrO}_{2}$ es estable en altas presiones parciales de $\mathrm{O}_{2}$, en casi todo el intervalo de las

Tabla II. Composición de la mezcla gaseosa de alimentación

Table II. Composition of the feeding gaseous mixture

\begin{tabular}{cccccc}
\hline Especie & $\begin{array}{c}\text { Flujo volumétrico } \\
\left(\mathrm{m}^{3} / \mathrm{s}\right)\end{array}$ & $\begin{array}{c}\text { Factor de compresibilidad }(\mathrm{Z}) \\
\left(78 \mathrm{KPa}, 25^{\circ} \mathrm{C}\right)\end{array}$ & $\begin{array}{c}\text { Flujo másico } \\
(\mathrm{g} / \mathrm{s})\end{array}$ & $\begin{array}{c}\text { Flujo molar } \\
(\mathrm{gmol} / \mathrm{s})\end{array}$ & $\begin{array}{c}\text { Fracción } \\
\mathrm{mol}\end{array}$ \\
\hline $\mathrm{Ar}$ & $8,33 \times 10^{-7}$ & 0,999546 & - & $2,62 \times 10^{-5}$ & 0,921 \\
$\mathrm{O}_{2}$ & $5 \times 10^{-8}$ & 0,998893 & - & $1,58 \times 10^{-6}$ & 0,064 \\
& $($ máximo $)$ & - & $1,96 \times 10^{-4}$ & $4 \times 10^{-7}$ & 0,015 \\
$\operatorname{Ir}(\mathrm{acac})_{3}$ & - & & $($ máximo $)$ & & \\
\hline
\end{tabular}



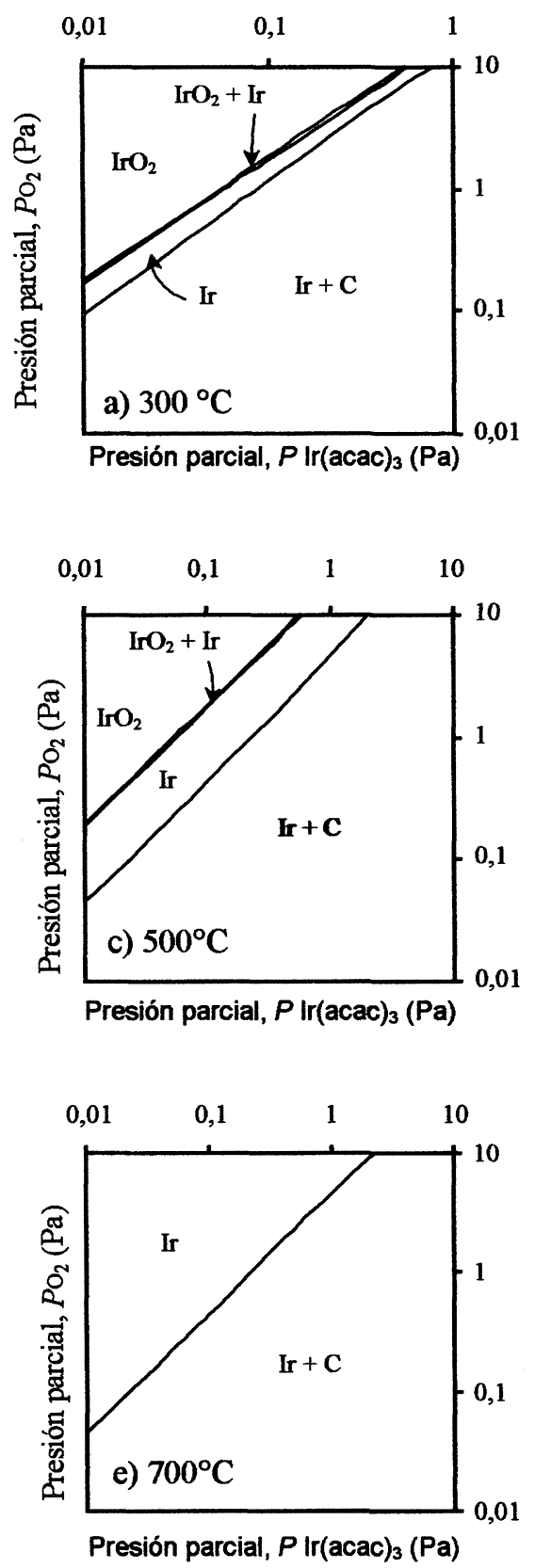
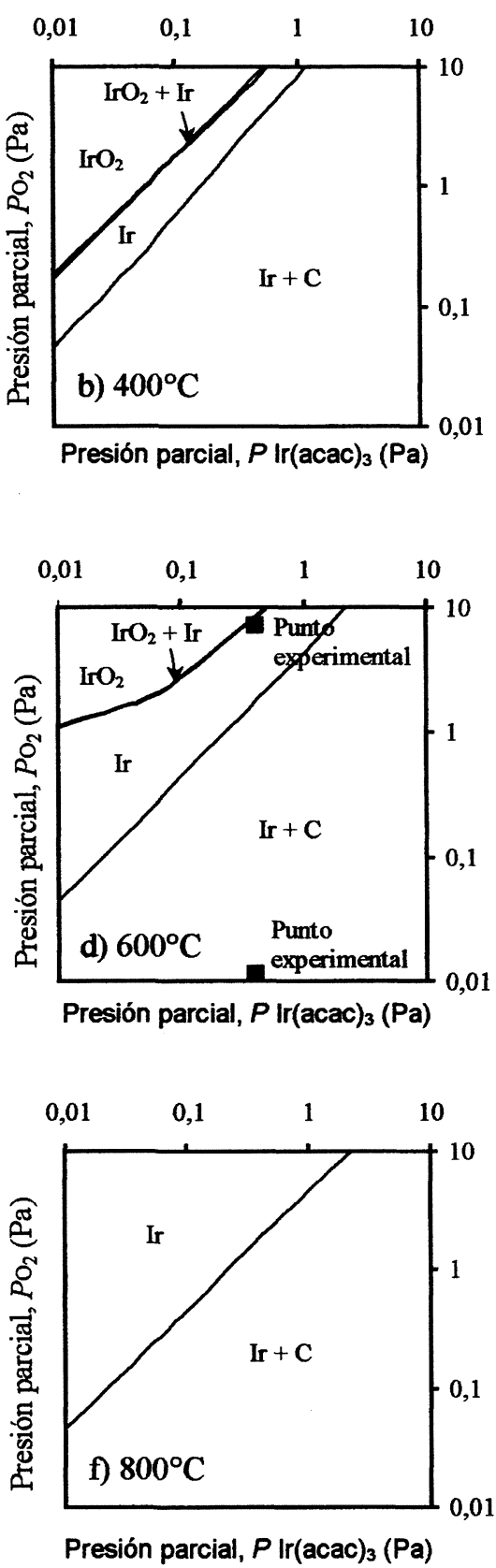

Figura 1. Diagramas de fase Ir-CVD para la mezcla gaseosa Ir(acac) ${ }_{3}-\mathrm{O}_{2}-\mathrm{Ar}$, para temperaturas en el rango $300-800^{\circ} \mathrm{C}$, calculados en función de las presiones parciales $(P)$ de $\operatorname{Ir}(\mathrm{acac})_{3}$ y $\mathrm{O}_{2}$ a presión total $P_{\text {tot }}=133,3 \mathrm{~Pa}$. Los puntos experimentales en la figura 1 (d) fueron obtenidos por Goto, Vargas y Hirai ${ }^{[6,7}$ y 12$]$.

Figure 1. Ir-CVD phase diagrams for Ir (acac) ${ }_{3}-\mathrm{O}_{2}-\mathrm{Ar}$ gaseous mixture calculated as a function of partial pressures $(P)$ of $\operatorname{Ir}(a c a c)_{3}$ and $\mathrm{O}_{2}$ at total pressure of $P_{\text {tor }}=133.3 \mathrm{~Pa}$ for temperatures between $300-800^{\circ} \mathrm{C}$. The experimental points in the fig. $l(d)$ were obtained by Goto, Vargas and Hirai ${ }^{[6,7}$ and 12$]$

presiones parciales de $\operatorname{Ir}(\mathrm{acac})_{3}$. Adyacente a esta fase se encuentra una banda de estabilidad para la mezcla de fases sólidas $\mathrm{IrO}_{2}+\mathrm{Ir}$. Enseguida, se puede observar otra banda más ancha para la fase sólida Ir. En la parte inferior derecha del diagrama se observa la región de estabilidad para las fases $\mathrm{I} r+\mathrm{C}$.
A temperaturas mayores, $T_{\text {dep }}=400,500$ y $600{ }^{\circ} \mathrm{C}$ (Figs. 1b-1d) se observan las mismas regiones de estabilidad. El área de la región monofásica $\mathrm{IrO}_{2}$ permanece aproximadamente constante hasta $T_{\text {dep }}$ $=500^{\circ} \mathrm{C}$. Lo mismo se observa para la región bifásica $\mathrm{IrO}_{2}+\mathrm{Ir}$. Sin embargo, el área de la fase $\mathrm{Ir}$ 
aumenta con la temperatura. Los diagramas IrCVD muestran que a $T_{\text {dep }}=700$ y $800^{\circ} \mathrm{C}$ (Figs. 1e y 1f) sólo aparecen la región monofásica Ir y la región bifásica $\mathrm{Ir}+\mathrm{C}$. La ausencia de la fase sólida $\mathrm{IrO}_{2}$ a $\mathrm{T}_{\text {dep }}>600^{\circ} \mathrm{C}$ está relacionada con valores más positivos de la energía libre estándar $\left(\Delta \mathrm{g}^{\circ}\right)$ de la reacción de oxidación de Ir con el incremento de temperatura. Esto es, a bajas temperaturas la fase más estable es $\mathrm{IrO}_{2}$, mientras que a temperaturas elevadas la fase más estable es Ir.

Las regiones de estabilidad mostradas en los diagramas de fases son significativamente importantes para las aplicaciones de las películas de Ir. Por ejemplo, para pistas conductoras y contactos útiles en la integración de circuitos electrónicos se requieren películas de Ir de alta pureza y calidad cristalina $^{[7]}$. En contraste, para electrodos aplicados a electrolitos sólidos, se requieren películas porosas constituidas por la mezcla de fases $\mathrm{Ir}+\mathrm{C}^{[7]}$.

En el diagrama calculado de la figura 1d) se muestran los resultados experimentales comunicados por Goto, Vargas y Hirai ${ }^{[6,7}$ y 12$]$. Estos investigadores prepararon películas de Ir por MOCVD a partir de $\operatorname{Ir}(\mathrm{acac})_{3}$ en los intervalos de $\mathrm{T}_{\text {dep }}=300$ $800{ }^{\circ} \mathrm{C}$ y $\mathrm{P}_{\text {tot }}=133,3-13.332 \mathrm{~Pa}$ con velocidades de depósito que varían de 0,01 a 0,4 nm/s. De acuerdo a sus análisis de espectroscopia electrónica Auger, ellos indican que las películas preparadas en ausencia de $\mathrm{O}_{2}$ a $\mathrm{T}_{\text {dep }}=600{ }^{\circ} \mathrm{C}$ y $\mathrm{P}_{\text {tot }}=133,3$ Pa presentan contaminación de carbono, es decir, las películas consisten de una mezcla de fases Ir+C. El punto inferior indicado en la figura 1d) es el resultado experimental de las películas preparadas sin la adición de $\mathrm{O}_{2}$, el cual es consistente con las fases predichas por el diagrama calculado. Por otra parte, la adición controlada de $\mathrm{O}_{2}$ promueve la obtención de películas de Ir puro. Los investigadores comunican la obtención de películas de Ir de alta pureza bajo las mismas condiciones de depósito arriba mencionadas y con la adición de $5 \times 10^{-8} \mathrm{~m}^{3} / \mathrm{s}$ de $\mathrm{O}_{2}$. Este resultado experimental representado por el punto superior en la misma figura, también es consistente con la predicción del diagrama de fases. Adiciones mayores de $\mathrm{O}_{2}$ generan la formación del compuesto $\mathrm{IrO}_{2}$ según los resultados experimentales de estos investigadores y la predicción del diagrama de fases.

El efecto de la temperatura de depósito y la adición de $\mathrm{O}_{2}$ a la mezcla gaseosa $\operatorname{Ir}(\mathrm{acac})_{3}-\mathrm{O}_{2}$ - $\mathrm{Ar}$ en la composición de las películas a $\mathrm{P}_{\text {tot }}=133,3 \mathrm{~Pa}$ y presión parcial de $\operatorname{Ir}(\mathrm{acac})_{3}$ de 0,1 Pa se presenta en la figura 2. La región bifásica $\mathrm{Ir}+\mathrm{C}$ se presenta en todo el intervalo de temperaturas $\mathrm{T}_{\mathrm{dep}}=300$ -

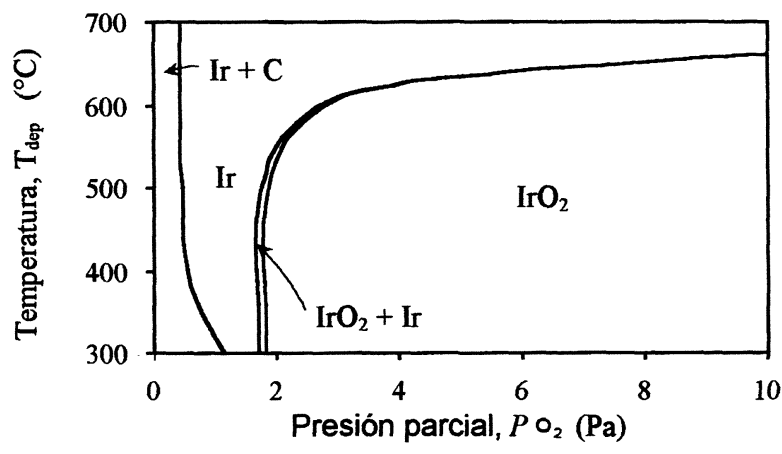

Figura 2. Diagrama de fase Ir-CVD para la mezcla gaseosa Ir (acac) $)_{3}-\mathrm{O}_{2}$-Ar calculado en función de la presión parcial de $\mathrm{O}_{2}$ y la temperatura de depósito a una presión total $P_{\text {tot }}$ $=133,3 \mathrm{~Pa}$ y presión parcial de $\operatorname{Ir}(\text { acac })_{3} P_{\text {Ir (acac) } 3}=0,1 \mathrm{~Pa}$.

Figure 2. Ir-CVD phase diagrams for $\operatorname{Ir}(a c a c)_{3}-\mathrm{O}_{2}-\mathrm{Ar}$ gaseous mixture calculated as a function of partial pressure of $\mathrm{O}_{2}$ and deposition temperature at total pressure of $P_{\text {tot }}=$ 133.3 Pa and partial pressure of Ir(acac $)_{3} P_{\text {Ir (acac }) 3}=0.1 \mathrm{~Pa}$.

$700{ }^{\circ} \mathrm{C}$ y bajas presiones parciales de $\mathrm{O}_{2}$. Esto implica que con pequeñas adiciones de oxígeno (o su total ausencia) en la mezcla gaseosa, se tendrán películas bifásicas de $\mathrm{Ir}+\mathrm{C}$ independientemente de la temperatura de depósito. La región monofásica de Ir tiene un intervalo estrecho a bajas presiones parciales $P_{\mathrm{O}_{2}} \sim 0,5-1,7$ y temperaturas de depósito $\mathrm{T}_{\mathrm{dep}}<600^{\circ} \mathrm{C}$. Sin embargo, a mayores temperaturas la región de estabilidad de Ir se extiende aún a altas presiones $P_{\mathrm{O}_{2}} \sim 1,7-10 \mathrm{~Pa}$. La región bifásica $\mathrm{IrO}_{2}+\mathrm{Ir}$ se presenta como una banda angosta. La región monofásica $\mathrm{IrO}_{2}$ tiene una extensión amplia entre $P_{\mathrm{O}_{2}} \sim 1,83-10$ Pa y $\mathrm{T}_{\text {dep }}<660{ }^{\circ} \mathrm{C}$.

La figura 3 muestra los resultados calculados de la variación de la composición de la fase gaseosa

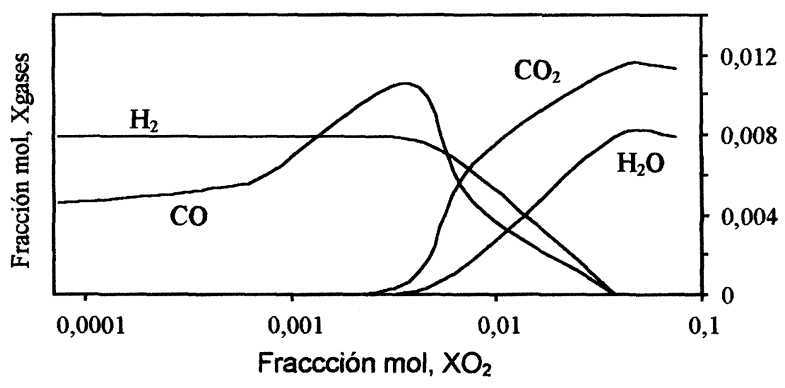

Figura 3. Variación de la composición de la fase gaseosa en función de la fracción mol de $\mathrm{O}_{2}$ alimentada en la mezcla gaseosa Ir (acac) $)_{3}-\mathrm{O}_{2}-\mathrm{Ar}$ a $\mathrm{T}_{\text {dep }}=500{ }^{\circ} \mathrm{C}, \mathrm{P}_{\text {tot }}=13,3 \mathrm{~Pa}$ y $P_{\text {Ir }(\text { acac }) 3}=0,01 \mathrm{~Pa}\left(X_{\text {Ir }(\text { acac }) 3}=7,5 \times 10^{-4}\right)$.

Figure 3. Variation of the gaseous phase composition as a function of $\mathrm{O}_{2}$ fraction mol feeding in the $\operatorname{Ir}(\text { acac })_{3}-\mathrm{O}_{2}-\mathrm{Ar}$ gaseous mixture at $T_{\text {dep }}=500{ }^{\circ} \mathrm{C}, P_{\text {tor }}=13.3 \mathrm{~Pa}$ y $P_{\text {Ir }(a c a c) 3}=$ $0.01 \mathrm{~Pa}\left(X_{\text {Ir }(a c a c) 3}=7.5 \times 10^{-4}\right)$. 
en función del $\mathrm{O}_{2}$ alimentado en la mezcla $\operatorname{Ir}(\mathrm{acac})_{3}-\mathrm{O}_{2}-\mathrm{Ar}$ a $\mathrm{T}_{\text {dep }}=500{ }^{\circ} \mathrm{C}, \mathrm{P}_{\text {tot }}=13,3 \mathrm{~Pa}$ y $P_{\mathrm{Ir}(\mathrm{acac})_{3}}=0,1 \mathrm{~Pa}$. La figura indica los principales productos gaseosos de la descomposición térmica del $\operatorname{Ir}(\mathrm{acac})_{3}$ tales como $\mathrm{CO}, \mathrm{H}_{2}, \mathrm{CO}_{2}$ y $\mathrm{H}_{2} \mathrm{O}$. Éstos son consistentes con las especies más significativas determinadas experimentalmente mediante espectrometría de masas para la descomposición del $\mathrm{Cu}(\text { acac })_{2}$ a $500{ }^{\circ} \mathrm{C}^{[14]}$.

La figura 4 muestra el efecto de la presión total y la temperatura de depósito en el contenido de C de las películas de Ir a partir de la mezcla gaseosa $\operatorname{Ir}(\mathrm{acac})_{3}$-Ar. En la figura se indican dos líneas de composición constante de 38 \% y $40 \%$ masa de C y una región localizada a altas temperaturas de composición constante del $36 \%$ masa de C. La disminución del contenido de carbono con la temperatura puede relacionarse con el incremento del $\mathrm{CO}$ en la fase gaseosa como lo indica la figura 5. La variación del contenido de $\mathrm{C}$ en la fase sólida, así como la variación de $\mathrm{CO}$ en la fase gaseosa en función de la temperatura de depósito a $\mathrm{P}_{\text {tot }}=$ $133,3 \mathrm{~Pa}$ y presión parcial de $\operatorname{Ir}(\mathrm{acac})_{3}$ de $0,012 \mathrm{~Pa}$ se ilustra en la figura 5. Los cálculos muestran que a presión total constante, el contenido de $\mathrm{C}$ en la fase sólida disminuye con la temperatura, en tanto que el contenido de $\mathrm{CO}$ en la fase gaseosa aumenta hasta un límite a partir del cual ambos contenidos permanecen constantes.

\section{CONCLUSIONES}

Se calcularon los diagramas de fase Ir-CVD empleando el método de minimización de la energía libre de Gibbs. Se exploraron condiciones típicas

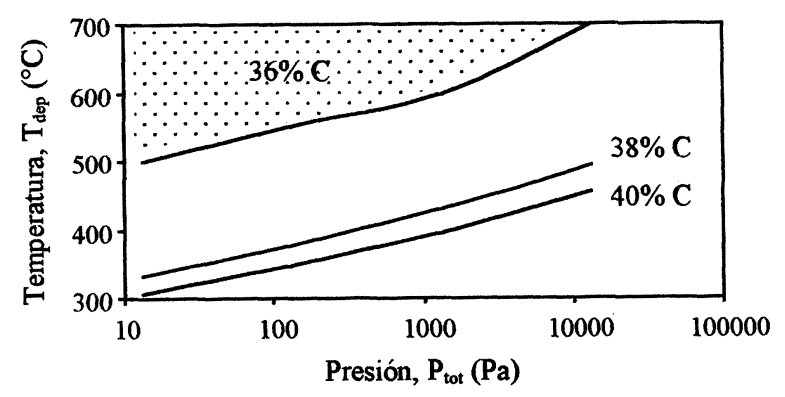

Figura 4. Efecto de la presión total y la temperatura de depósito en el contenido de carbono de las películas de Ir a partir de la mezcla gaseosa $\operatorname{Ir}(a c a c)_{3}-A r$. $X_{1 r(a c a c) 3}=$ $7,5 \times 10^{-4}$.

Figure 4. Effect of the total pressure and deposition temperature on the content of carbon in the Ir films using Irlacac) ${ }_{3}$-Ar gaseous mixture. $X_{\text {Ir (acac) } 3}=7.5 \times 10^{-4}$.

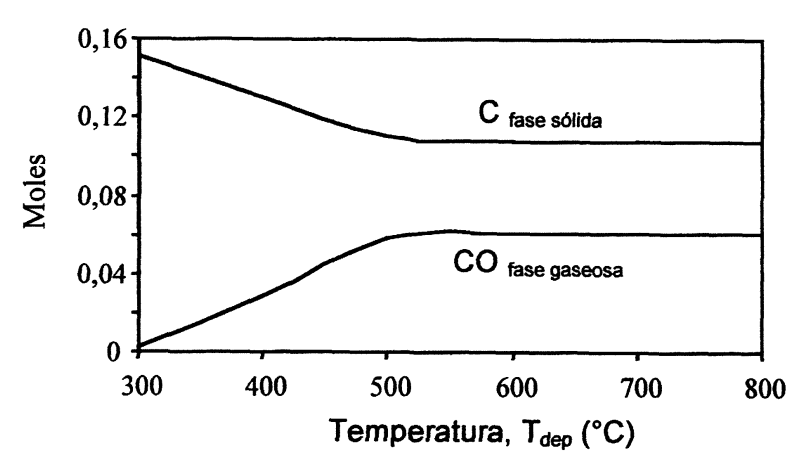

Figura 5. Contenido de $\mathrm{C}$ en la fase sólida y $\mathrm{CO}$ en la fase gaseosa en función de la temperatura de depósito para la mezcla gaseosa $\operatorname{Ir}(\text { acac })_{3}-A r$ a $P_{\text {tot }}=133,3 \mathrm{~Pa}$ y $P_{\text {Ir (acac) } 3}=$ $0,012 \mathrm{~Pa}$

Figure 5. Content of $\mathrm{C}$ in the solid phase and $\mathrm{CO}$ in the gaseous phase as a function of deposition temperature for the $\operatorname{Ir}(\text { acac })_{3}-A r$ gaseous mixture at $P_{\text {tot }}=133.3 \mathrm{~Pa}$ and $P_{\text {Ir }(\text { acac } 33}=0.012 \mathrm{~Pa}$.

para la preparación de películas de Ir tales como $\mathrm{T}_{\text {dep }}=300-800{ }^{\circ} \mathrm{C}$ y $\mathrm{P}_{\text {tot }}=13,3-13.332 \mathrm{~Pa}$. Se investigó el efecto de la adición de $\mathrm{O}_{2}$ en la composición química de las películas. Los diagramas permiten elegir las condiciones apropiadas de depósito $\left(\mathrm{T}_{\text {dep }}, \mathrm{P}_{\text {tot }}\right.$ y $\left.P_{\mathrm{i}}\right)$ para obtener películas de la composición deseada y predicen que en ausencia de $\mathrm{O}_{2}$, las películas constarán de $\mathrm{Ir}+\mathrm{C}$, independientemente de la temperatura de depósito y la presión total dentro del reactor. Las películas formadas por $\mathrm{Ir}+\mathrm{C}$ tendrán como mínimo un $36 \%$ masa de C. La adición controlada de $\mathrm{O}_{2}$ promueve la formación de películas de Ir puro. El diagrama Ir-CVD calculado para la mezcla gaseosa $\operatorname{Ir}(\mathrm{acac})_{3}$ $\mathrm{O}_{2}$-Ar, a $\mathrm{T}_{\text {dep }}=600^{\circ} \mathrm{C}$ y $\mathrm{P}_{\text {tot }}=133,3 \mathrm{~Pa}$, predice apropiadamente las fases presentes en las películas de Ir obtenidas en trabajos experimentales reportados en la literatura.

\section{Agradecimientos}

Este estudio se realizó con el apoyo financiero otorgado a través del proyecto IPN-CGPI-200812. Los autores agradecen a CONACYT, SNI, COFFA e IPN-PIFI las becas otorgadas.

\section{REFERENCIAS}

[1] J.H. Kwong y S.G. YoOn, J. Electrochem. Soc. 1448 (1997).

[2] B.S. Kwak, P.N. First y A. ERbil, J. Appl. Phys. 72, 8 (1992) 3735-3740. 
[3] Dossi, R. Psaro y A. Bartsch, Catal. Today 17 (1993) 527-535.

[4] R. Feurer, A. Reynes et al, J. Phys. IV 5 C5 (1995) $1037-$ 1043.

[5] W. Zhang, R. Vargas y T. Goto, Appl. Phys. Lett. 64, 11 (1994) 1359-1361.

[6] R. Vargas, T. Goto y W. Zhang, Appl. Phys. Lett. 65, 9 (1994) 1094-1096.

[7] T. Goto, J.R. Vargas y T. Hirai, Mater. Sci. Eng. A217 218 (1996) 223-226.

[8] M.L. Hitchman y K.F. Jensen, Chemical Vapor Deposition, principles and applications, Ed. Academic Press Limited, Gran Bretaña, 1993, pp. 8-12.

[9] C. Bernard, M. Pons et al, MRS Bull. (1999) 27-31.

[10] V.G. Bessergenev, N.V. Gelfond y I.K. Igumenov, Supercond. Sci. Technol. 4 (1991) 273-277.

[11] D.C. Smith, S.G. Pattillo, N.E. Elliot y T.G. Zocco, Mat. Res. Soc. Symp. Proc. 168 (1990).

[12] T. Goto, J.R. VARgas y T. HiRAI, Mater. Trans. JIM 40, 3 (1999).
[13] C.W. Bale, A.D. Pelton y W. T.Thompson, FACT-WinUser Manual CRCT, Ecole Polytechnique de Montreal, Canadá, 1999, www.crct.polymtl.ca.

[14] A.E. Turgambaeva, A.F. Bykov e I.K. Igumenov, Thermochim. Acta 256 (1995).

[15] F.R. Lang y K.H. Dahmen, Thin Solid Films 241 (1994).

[16] J.V. Hoene, R.G. Charles y W.M. Hickam, J. Phys. Chem. 62 (1958) 1.098-1.101.

[17] S.W. Benson, Thermochemical Kinetics, Ed. John Wiley \& Sons, Inc., Nueva York, EE.UU., 1968, pp.178-193.

[18] J.M. Hacking y G. Pilcher, J. Chem. Thermodyn. 11 (1979) 1.015-1.017.

[19] R.C. Reid y T.K. Sherwood, Propiedades de los gases y líquidos, Ed. UTEHA, $1^{\text {a }}$ ed. en español, México, 1968, pp. 189-200.

[20] R.C. Perry y C.H. Chilton, Chem. Eng. Handb., Ed. Mc. Graw Hill, $5^{\text {a }}$ ed., EE.UU., 1976, pp. 3/235-3/237. 